Journal of Applied Fluid Mechanics, Vol. 6, No. 1, pp. 143-148, 2013. Available online at www.jafmonline.net, ISSN 1735-3572, EISSN 1735-3645. DOI: $10.36884 / j a f m .6 .01 .19508$

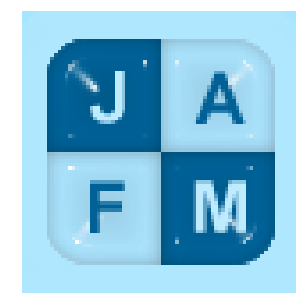

\title{
Bingham Fluid Flow through a Tapered Tube with Permeable Wall
}

\author{
K. Avinash ${ }^{1}$, J. Ananda Rao ${ }^{1}$, Y. V. K. Ravi $\operatorname{Kumar}^{2 \dagger}$ and S. Sreenadh ${ }^{3}$ \\ ${ }^{1}$ Department of Mathematics, Osmania University, Hyderabad, India \\ ${ }^{2}$ Practice School Division, Birla Institute of Technology and Science (BITS) - Pilani, Rajasthan, India \\ ${ }^{3}$ Department of Mathematics, Sri Venkateswara University, Hyderabad, India
}

†Corresponding Author Email: yvkravi@gmail.com

(Received March 29, 2011; accepted July 12, 2011)

\begin{abstract}
Bingham fluid flow through a tapered tube with permeable wall studied. The flow takes place due to pressure gradient, and the porous medium is homogeneous with permeability $\mathrm{k}$. The flow surrounded by the porous medium is governed by the Bingham model, and the flow in the porous medium is governed by the Darcy's law. The velocity distribution, the volume rate of flow and its fractional increase are obtained. The results are deduced and discussed through graphs.
\end{abstract}

Keywords: Bingham fluid, Tapered tube, Permeable wall.

\section{INTRODUCTION}

The blood vessel can be idealized as a tube with tissue space as circular porous bed (Vide Guha and Chuadhury, 1985).The rotating viscometer data of Rand et al. (1946), Bugliarello et al. (1965) and Chien et al. (1965) suggests the non-Newtonian behaviour of blood. Lew et al. (1971) suggested chyme as a non-Newtonian material having plastic-like properties. In view of this the biofluid flow in a living body can possess the non-Newtonian behaviour in general. In order to have a better understanding of blood flow in arteries and veins and chyme flow in stomach. It is necessary to consider the biofluid to be a yield stress fluid. One of the models for yield stress fluids is Bingham model. Hence the study of Bingham fluid flow through a tapered tube with permeable wall is of considerable importance in medicine.

Bird et al. (1960) investigated the Bingham fluid flow in a rigid circular tube. Rathy (1976) studied the flow of a Bingham fluid in a channel and in an annulus with impermeable walls. Vajravelu et al. (1987) made a study on the Bingham fluid in a circular tube with permeable wall. The velocity field is obtained using Beavers and Joseph (1967) slip condition at the permeable wall. Buckingham-Reiner equation for the flow is obtained.

The Bingham fluid flow between two permeable beds is discussed by Goverdhan et al. (1991). The flow in the channel is assumed to be governed by Bingham model.
The flow in the permeable beds is governed by Darcy's law. The velocity distribution is obtained. Some results are deduced and discussed. Comparini (1992) discussed a one-dimensional model for the time dependent flow of a Bingham fluid between two parallel plates. The global existence and uniqueness of classical solution to the problem is proved.

Ravana et al. (1996) studied the free surface flow of a Bingham fluid in an inclined channel over a permeable bed. The flow in the channel is described by Bingham model, whereas the flow in the permeable bed is according to Darcy's law. The velocity field, the shear stress, the mass flow rate and its fractional increase are obtained.

The problem of rotational motion of a Bingham fluid in the gap between two coaxial cylinders, the outer one being at rest and the inner one moving at given angular velocity is solved by Comparini (1997).

Narahari (1999) discussed unsteady flow of a Bingham fluid between two permeable beds having different permeabilities. The velocity distribution in the porous and non-porous regions is obtained. Some deductions are made and the results are discussed.

Sankara Reddy et al. (2000) made a detailed study on the Bingham fluid flow in an inclined channel bounded by two permeable beds. The velocity distribution in the 
porous and non-porous regions is obtained. The temperature variation with Ec.Pr is discussed.

Viswanatha Reddy et al. (2001) made a study on Bingham fluid flow in an annulus. The velocity field, the mass flow rate and its fractional increase are obtained. The results are deduced and discussed. Helical flow of a power-law fluid in a thin annulus with permeable walls is investigated by Vajravelu et al. (2003). It is observed that velocity increases due to permeable nature of the annulus.

In this paper, Bingham fluid flow through a tapered tube with permeable wall is investigated. The velocity distribution, the volume rate of flow and its fractional increase are obtained. The results are deduced and discussed through graphs.

\section{MAthematical Formulation OF THE Problem}

Consider the flow of a Bingham fluid through a tapered tube of length $\mathrm{L}$ with permeable wall. The flow takes place due to pressure gradient, and the porous medium is homogeneous with permeability $\mathrm{k}$. The flow surrounded by the porous medium is governed by the Bingham model, and the flow in the porous medium is governed by the Darcy's law.

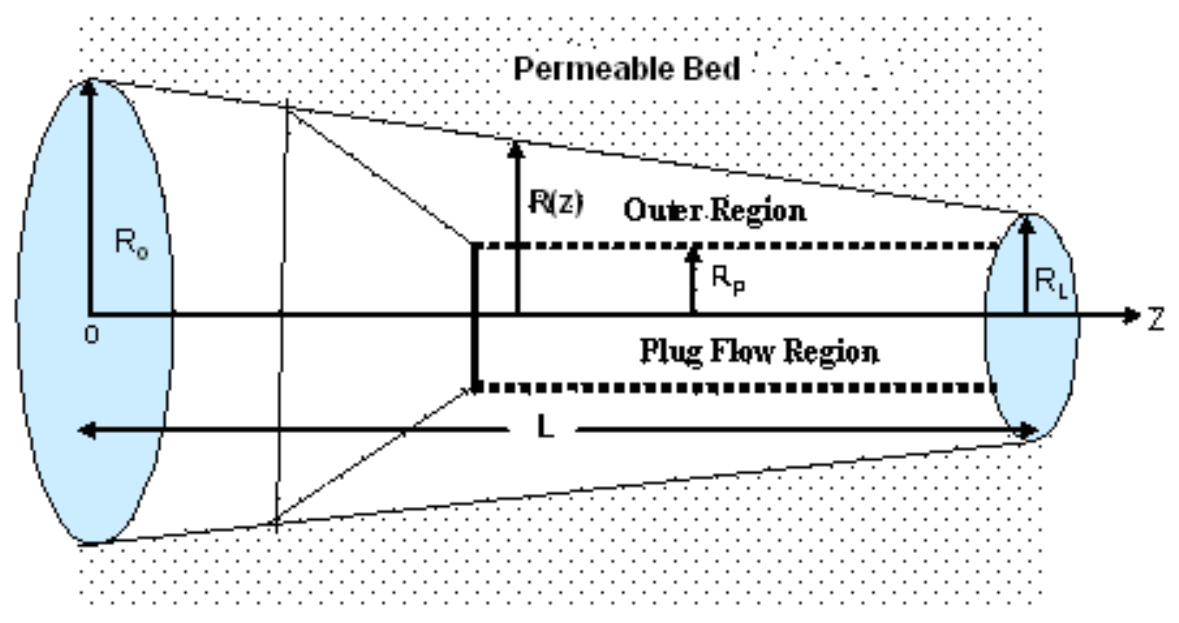

Fig.1. Physical Model

The tube has a radius $R_{o}$ at the entrance and radius $R_{L}$ at the exit. The tube radius at any distance $\mathrm{z}$ from the inlet is given by

$$
R(z)=R_{o}+\left(\frac{R_{L}-R_{o}}{L}\right) z
$$

The flow is axi-symmetric. Cylindrical polar coordinate system is used. The following assumptions are made in deriving the basic equations:

a) The flow is steady and incompressible

b) The flow is in axial direction.

c) All physical quantities except the pressure are function of $r$ only.

d) The body forces are negligible.

In view of the above assumptions, the basic equations and boundary conditions of the flow take the following form:

\section{Basic Equations}

$$
\begin{aligned}
& \frac{1}{r} \frac{d}{d r}\left(r \tau_{r z}\right)=-\left(\frac{d p}{d z}\right) \\
& \text { Where } \tau_{r z}=-\mu_{o} \frac{d v_{z}}{d r}+\tau_{o}
\end{aligned}
$$

\section{Boundary Conditions}

$$
\begin{aligned}
& \tau_{r z}=\text { finite at } r=0 \\
& \frac{d v_{z}}{d r}=0 \quad \text { at } \quad r=R_{p} \\
& v_{z}=u_{B} \quad \text { at } \quad r=R
\end{aligned}
$$

$\frac{d v_{z}}{d r}=\frac{\alpha}{\sqrt{k}}\left(u_{B}-Q\right) \quad$ at $\quad r=R$

where $Q=-\frac{k}{\mu_{o}}\left(\frac{d p}{d z}\right)$

Integrating (2) w.r.to ' $r$ ' and using the condition (4), we obtain

$\tau_{r z}=-\left(\frac{d p}{d z}\right) \frac{r}{2}$

Substituting (9) in (3), we get

$\tau_{o}-\mu_{o} \frac{d v_{z}}{d r}=-\left(\frac{d p}{d z}\right) \frac{r}{2}$ 
where $\tau_{o}, \tau_{r z}, \alpha, k, u_{B}, Q, \mu_{o}, u_{a v}, v_{z}, p$ and $R_{p}$ are the yield stress, Shear stress, slip parameter, permeability, slip velocity, Darcy velocity, coefficient of Viscosity at the interface $r=0$, average velocity, velocity component in $z$ - direction, pressure and radius of the plug flow region.

\section{Non-Dimensionalisation of the Flow Quantities}

$$
\begin{aligned}
& v_{z}^{*}=\frac{v_{z}}{u_{a v}} \quad u_{B}^{*}=\frac{u_{B}}{u_{a v}} \quad \tau_{o}^{*}=\frac{\tau_{o}}{\left(\frac{\mu_{o} u_{a v}}{R_{o}}\right)} \\
& \tau_{r z}^{*}=\frac{\tau_{r z}}{\left(\frac{\mu_{o} u_{a v}}{R_{o}}\right)} \quad Z^{*}=\frac{Z}{L} \quad P^{*}=\frac{P}{\left(\frac{L \mu_{o} u_{a v}}{R_{o}^{2}}\right)} \\
& R_{p}^{*}=\frac{R_{p}}{R_{o}} \quad r^{*}=\frac{r}{R_{o}} \quad R^{*}(z)=\frac{R(z)}{R_{o}} \\
& R^{*}(z)=\frac{R(z)}{R_{o}} \quad Q^{*}=\frac{Q}{u_{a v}}
\end{aligned}
$$

In view of the above dimensionless quantities the equations (1), (4) - (8) ) and (10) take the following form. The asterisks $(*)$ are omitted here after.

$R=1+\left(R_{L}-1\right) z$

$\frac{\tau_{o}}{r}-\frac{1}{r} \frac{d v_{z}}{d r}=\frac{P}{2}$, Where $P=-\frac{d p}{d z}$

$\frac{d v_{z}}{d r}=0 \quad$ at $\quad r=R_{p}$

$v_{z}=u_{B} \quad$ at $\quad r=R$

$$
\frac{d v_{z}}{d r}=\frac{\alpha}{\sqrt{k}}\left(u_{B}-D a P\right) \quad \text { at } \quad r=R
$$

where $D a=\frac{k}{R_{o}^{2}}$

\section{Solution of the Problem}

Solving (12) and using the boundary condition (14) we obtain the velocity field around the plug flow region as

$v_{1}=-\frac{P}{4}\left(r^{2}-R^{2}\right)+\tau_{o}(r-R)+u_{B}$

When $R_{p} \leq r \leq R$

Using (15) in (16), we get the slip velocity at the porous wall as

$u_{B}=\frac{P \sqrt{D a}}{\alpha}\left[\alpha \sqrt{D a}-\frac{R}{2}+\frac{\tau_{o}}{P}\right]$

Using (13) in (16), we obtain the relation between $\tau_{o}$ and $R_{p}$ as
$R_{p}=\frac{2 \tau_{o}}{P}$

Taking $r=R_{p}$ in equation (16) and using the relation (18), we get the velocity field in the plug flow region as

$v_{2}=\frac{P}{4}\left(R-R_{p}\right)^{2}+u_{B} \quad$ When $0 \leq r \leq R_{p}$

The flow in the porous region $(r \geq R)$ is governed by the Darcy law which is given by $Q=P . D a$

\section{DEDUCTIONS}

Case (i): When $\mathrm{R}(\mathrm{z})=1$ and the permeability parameter $k \rightarrow 0$ in the equations(16), (17) and (19) we get the velocity field outside the plug flow region $r \geq R_{p}$ :

$v_{1}=-\frac{P}{4}\left(r^{2}-1\right)+\tau_{o}(r-1)+u_{B}$

and that in the plug flow region $r \leq R_{p}$ :

$v_{2}=\frac{P}{4}\left(1-R_{p}\right)^{2}$

which are in agreement with those of Bird et al. (1960).

Case (ii): When $\mathrm{R}(\mathrm{z})=1$, the equations (16), (17) and (19) we get the velocity field for the flow of a Bingham fluid flow through a circular pipe with permeable wall as follows.

$v_{1}=-\frac{P}{4} r^{2}+\tau_{o} r+u_{B}+\frac{P}{4}-\tau_{o}$

When $R_{p} \leq r \leq 1$

$u_{B}=\frac{P \cdot \sqrt{D a}}{\alpha}\left[\alpha \cdot \sqrt{D a}-\frac{1}{2}+\frac{\tau_{o}}{P}\right]$

$v_{2}=\frac{P}{4}\left(1-R_{p}\right)^{2}+u_{B}$, when $r \leq R_{p}$

which agrees with the corresponding ones of Vajravelu and Sreenadh (1987).

Case (iii): When $\mathrm{R}(\mathrm{z})=1$ and as $\tau_{o} \rightarrow 0$, the velocity field for the flow with permeable wall is given by

$v_{z}=-\frac{P}{4} r^{2}+u_{B}+\frac{P}{4} \quad$ When $R_{p} \leq r \leq 1$

$u_{B}=\frac{P \cdot \sqrt{D a}}{\alpha}\left[\alpha \cdot \sqrt{D a}-\frac{1}{2}\right]$

These results are in agreement with the results of Sreenadh and Arunachalam (1983).

\section{VOLUME RATE OF FLOW}

The Volume rate of flow for the flow of a Bingham fluid flow through a tapered tube is 
$Q^{1}=\int_{0}^{2 \pi} \int_{0}^{R_{p}} v_{2} r d r d \theta+\int_{0}^{2 \pi} \int_{0}^{R} v_{1} r d r d \theta$

$Q^{1}=\frac{p \pi}{8}\left(B_{1}\right)-\frac{\pi \tau_{0}}{3}\left(B_{2}\right)+u_{B} \pi R^{2}$

Where $\quad B_{1}=R^{4}+3 R_{p}^{4}-4 R R_{p}^{3} \quad$ and

$B_{2}=R^{3}-2 R_{p}^{3}+3 R R_{p}^{2}$

When the permeability parameter $k \rightarrow 0($ i.e., $D a \rightarrow 0)$ (28) reduces to

$Q_{0}^{1}=\frac{P \pi}{8}\left(B_{1}\right)-\frac{\pi \tau_{0}}{3}\left(B_{2}\right)$

Where $B_{1}=R^{4}+3 R_{p}^{4}-4 R R_{p}^{3}$

and $B_{2}=R^{3}-2 R_{p}^{3}+3 R R_{p}^{2}$

\section{FRACTIONAL INCREASE}

The fractional increase in the volume rate of flow of the Bingham fluid through a tapered tube with permeable wall over what it would be if the wall of the tube were impermeable is

$$
F=\frac{\left(Q^{1}-Q_{0}^{1}\right)}{Q_{0}^{1}}=\frac{u_{B} R^{2}}{\left(\frac{P B_{1}}{8}-\frac{\tau_{o} B_{2}}{3}\right)}
$$

where $B_{1}=R^{4}+3 R_{p}^{4}-4 R R_{p}^{3} \quad$ and $B_{2}=R^{3}-2 R_{p}^{3}+3 R R_{p}^{2}$

\section{DisCuSSION OF THE RESULTS}

The Velocity profiles are shown in figures (1) - (11) for different values of $\tau_{0}$, Darcy number $\mathrm{Da}, \quad P=10$ and $\alpha=1$. It is observed that the velocity attains maximum value at $r=0$ and decreases with the increment in $\mathrm{r}$.

For fixed $r$ and $\mathrm{Da}$, the velocity decreases due to increase in z. The slip velocity at the permeable wall decreases with the increment in the value of Da. On comparing the velocity profiles for permeable and impermeable tapered tubes, it is found that the velocity is enhanced due to the permeability of the wall of the tapered tube. It is also observed that the velocity remains constant from the axis (i.e., $r=0$ ) up to some value of $r$ (which is the Plug flow region) and then decreases to a value at the permeable wall (which is the non Plug flow region).

For fixed $r$ and $\tau_{\mathrm{o}}$, the velocity increases with decrement in Da. As Da decreases the gap between the velocity curves becomes smaller for any fixed $\tau_{\mathrm{o}}$. For larger $\tau_{\mathrm{o}}$, there is an increase in plug flow region. From Fig (12) it is observed that, for the fixed Darcy number Da, the velocity decreases along the axis with the increase in $\tau_{\mathrm{o}}$.It is the other way at the permeable wall.

The fractional increase in volume rate of flow is numerically evaluated and is depicted in Fig (13). It is observed that for a fixed Darcy number Da, the fractional increase increases with yield stress $\tau_{\mathrm{o}}$.

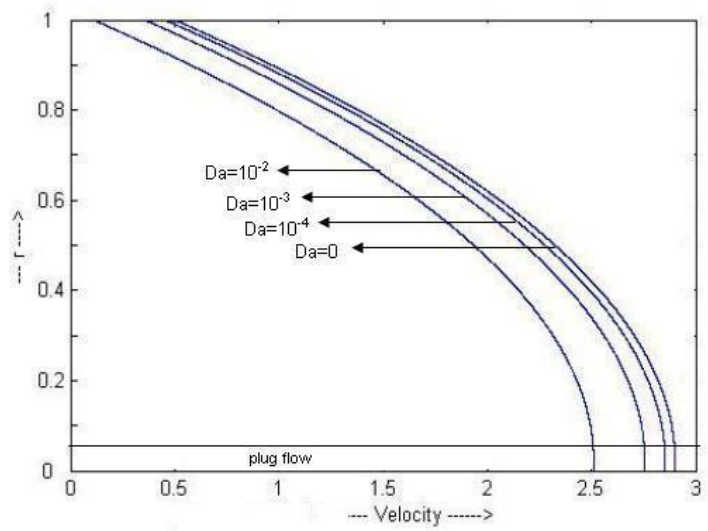

Fig. 1. Velocity profiles for $\mathrm{z}=0, \tau_{0}=0.1$

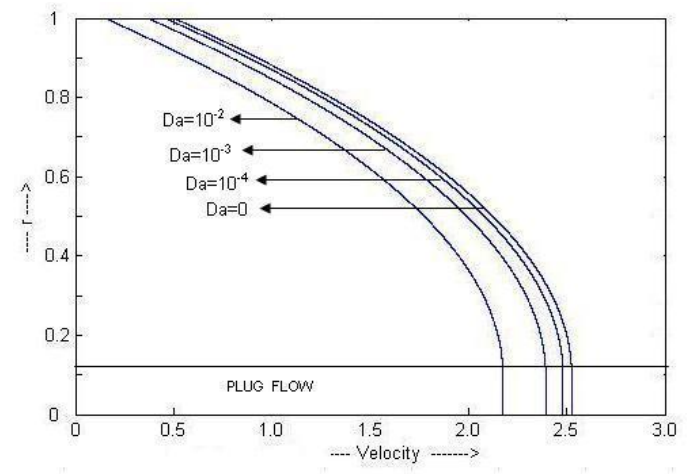

Fig. 2. Velocity profiles for $z=0, \tau_{0}=0.5$

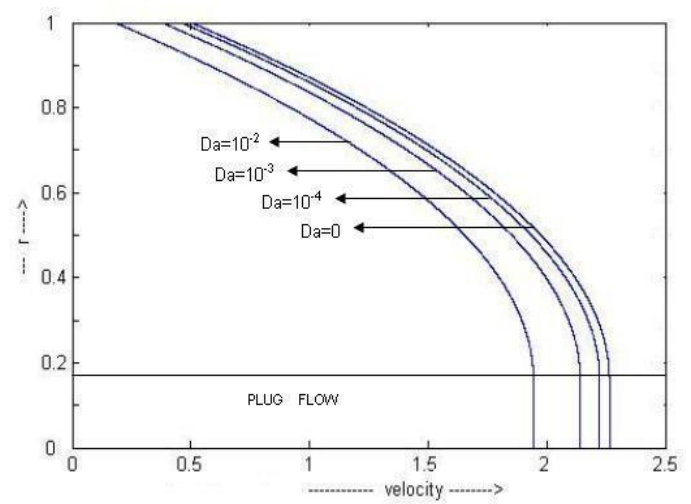

Fig. 3. Velocity profiles for $z=0, \tau_{0}=0.8$ 


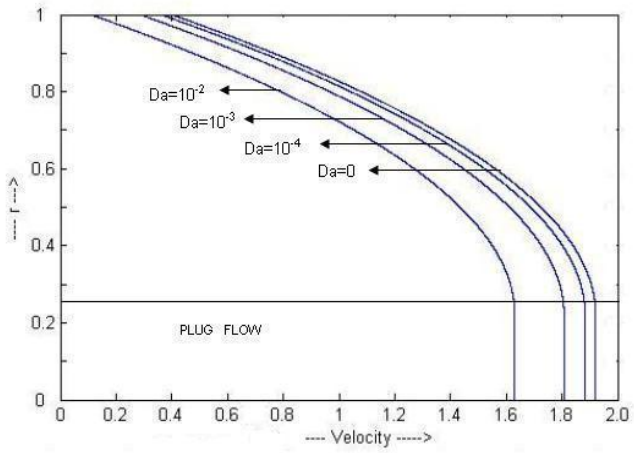

Fig. 4. Velocity profiles for $\mathrm{z}=0, \tau_{0}=1.1$.

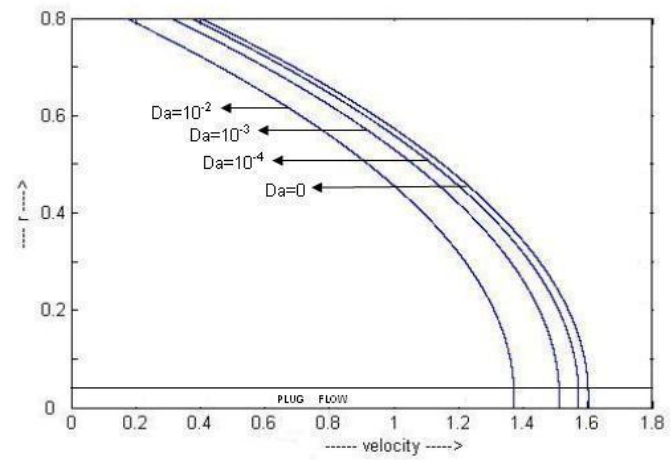

Fig. 5. Velocity profiles for $\mathrm{z}=0.4, \tau_{0}=0.1$.

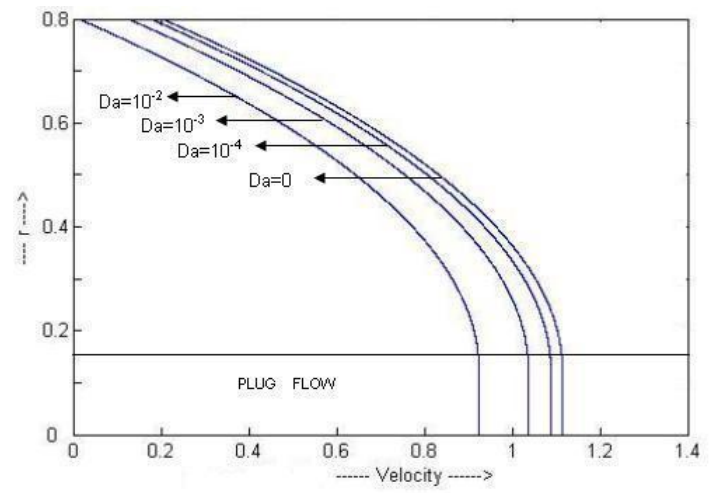

Fig. 6. Velocity profiles for $\mathrm{z}=0.4, \tau_{0}=0.5$.

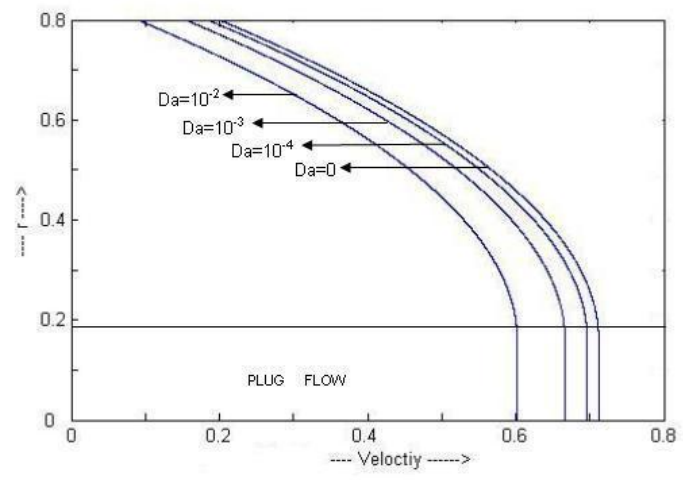

Fig. 7. Velocity profiles for $\mathrm{z}=0.4, \tau_{0}=0.8$.

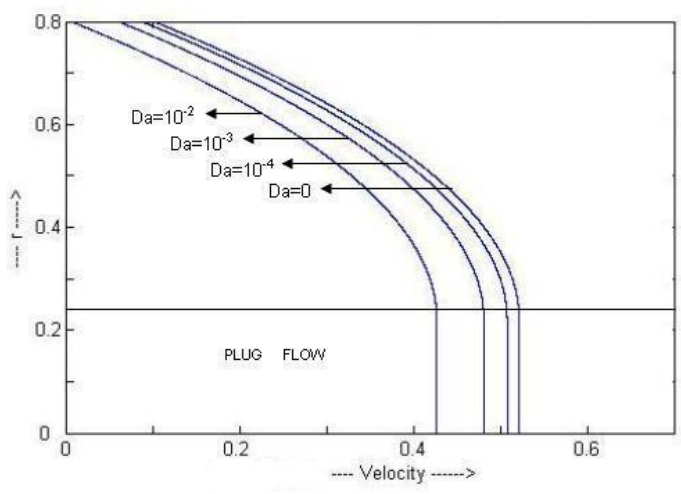

Fig. 8. Velocity profiles for $\mathrm{z}=0.4, \tau_{0}=1.1$.

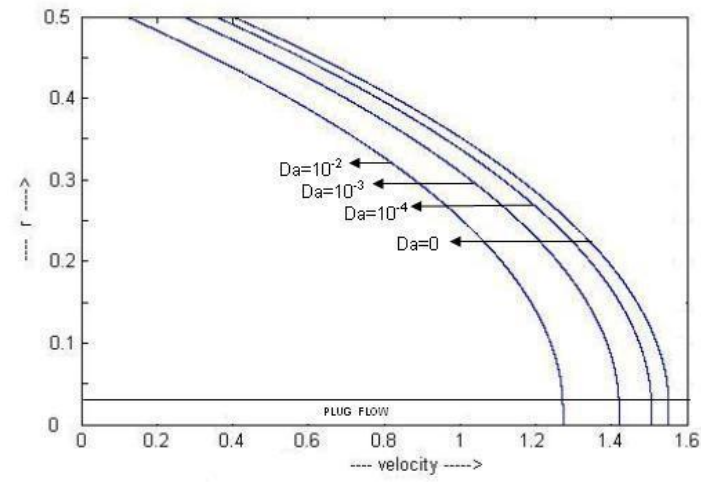

Fig. 9. Velocity profiles for $\mathrm{z}=1, \tau_{0}=0.1$.

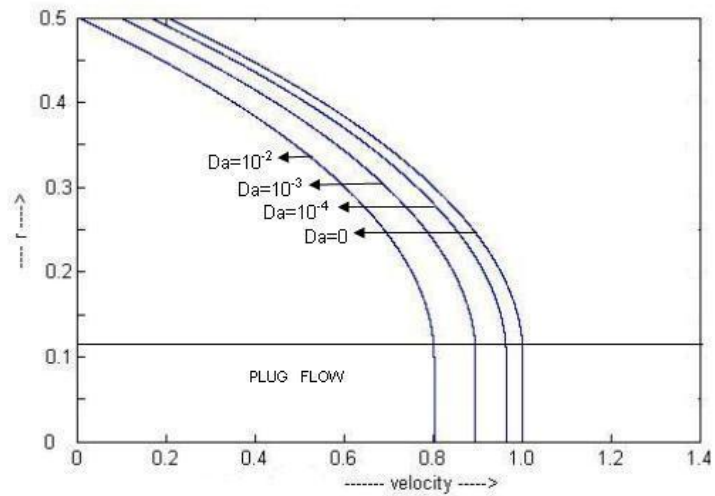

Fig. 10. Velocity profiles for $\mathrm{z}=1, \tau_{0}=0.5$.

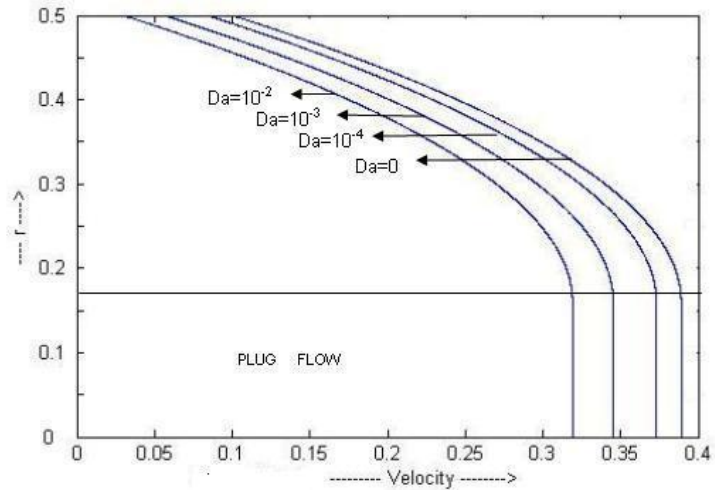

Fig. 11. Velocity profiles for $\mathrm{z}=1, \tau_{0}=0.8$ 


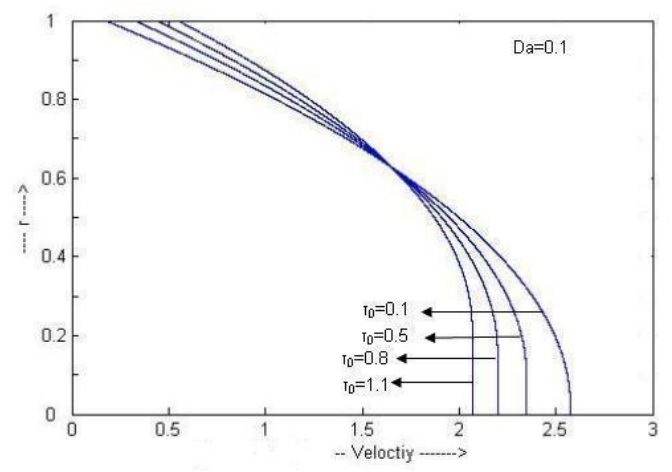

Fig. 12. Velocity profiles for $\mathrm{Da}=0.1$

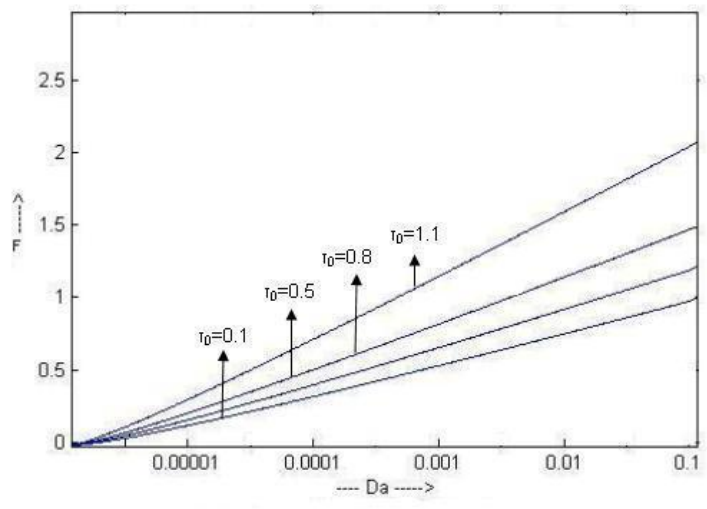

Fig. 13. Fractional increase.

\section{REFERENCES}

P.W.Rand, E.Lacombe, H.E.Hunt and W.H.Austin, 1964 Viscosity of normal blood under Normothermic and Hypothermic conditions. J. Appl. Physiol. 19, 117122.

G.Bugliarello, C.Kapur and G.Hsiao, 1965 Proceedings of the fourth International Congress on Rheology, 4, Symposium of Biorheology (Edited by Copley, A.L.), Inter Sciences New York, 351.

S.Chien, S.Usami, H.M.Taylor, J.L.Lundberg and M.T.Gregre-Sons, 1965 Effects of haemotocrit and plasma proteins on human blood Rheology at low shear rates. J. Appl. Phys. 21, 81-87.

H.S.Lew, Y.C.Fung and C.B.Lowenstein, 1971 Peristaltic carrying and mixing of chyme in small intestine. $J$. Biomechanics. 4, 297-315.
R.B.Bird, W.E.Stewart and E.N.Light Foot, 1960 Transport Phenomena. John Wiley \& Sons. Inc. New York.

R.K.Rathy, 1976 An Introduction to Fluid Dynamics. Oxford and IBH Publishing Co., New Delhi, Bombay, Calcutta.

K.Vajravelu, S.Sreenadh, S.Ramakrishna and P.V.Arunachalam, 1987 Bingham fluid flow through a circular pipe with permeable wall. ZAMM. 67, 568-569.

G.S.Bervers and D.D.Joseph, 1967 Boundary conditions at a naturally permeable wall. J. Fluid Mech. 30, 197-207.

S.Goverdhan, S.Sreenadh and P.V.Arunachalam, 1991 Bingham fluid flow between two permeable beds. Proc. Nat. Acad. Sci. India. 61A, 19-27.

E.Comparini, E. 1992 A one-dimensional Bingham flow. Journal of Mathematical Analysis and Applications. $169,127-139$.

G.Ravana S.Ramakrishna, S.Sreenadh and P.V.Arunachalam, 1996 Bingham fluid flow through an inclined channel over a permeable bed. Bulletin of Pure and Applied Sciences. 15E, No.1, 47-60

E.Comparini, 1997 Regularization procedures of singular free boundaries problems in rotational Bingham flows. ZAMM. 77. No.3, 1-12.

M.Narahari, S.Sreenadh, P.V.Arunachalam, P.V. 1999 Unsteady flow of a Bingham fluid between two permeable beds. J. Indian Acad. Math. 22, No.2, 225-242.

P.Sankara Reddy, S.Sreenadh and P.V.Arunachalam, 2000 Bingham fluid flow in an inclined channel bounded by two permeable beds. J. Indian Acad. Math. 22, No.1, 143-162.

G.Viswanatha Reddy, S.Sreenadh, P.V.Arunachalam, 2001 Bingham fluid flow in an annulus.J. Indian Acad.Math. 23, No.2.

K.Vajravelu, K.ramesh, S.Sreenadh and P.V.Arunachalam, 2003 pulsatile flow between permeable beds. Int. J.Of Non-linear Mechanics.38, 999-1005. 